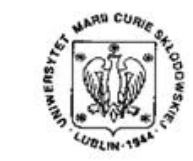

Annales UMCS Informatica AI X, 1 (2010) 69-78 DOI: $10.2478 / \mathrm{v} 10065-010-0037-5$

\section{Annales UMCS}

Informatica

Lublin-Polonia

Sectio AI

http://www.annales.umcs.lublin.pl/

\title{
Image quality index of the monochrome archival photographs' compression
}

\author{
Marzena Gromaszek*, Mariusz Michalik ${ }^{\dagger}$ \\ Humanistic and Economics University, \\ ul. Rewolucji 1905 r. nr 64, 90-222 Eódź, Poland.
}

\begin{abstract}
The recent process of digitalizing archives has increased the importance of choosing the best compression method and evaluating the quality of the compressed materials. Our paper focuses on monochrome photographs. We suggest a new image quality index partly based on Human Visual System. We think that, despite its simplicity, it is equal to Mean Subjective Rank. In addition, we intend to ascertain that (the index submitted by us) our index is very easy both to understand and to implement.
\end{abstract}

\section{Introduction}

The evaluation of digital photographs has an integral part in many relevant processes, especially so in the process of digitalizing of the archive materials, such as books, documents, photographs. Undoubtedly, the quality of these materials should be optimized according to preferences and expectations of the archives potential users. However, fulfilling this criterion would generate many obstacles in practice [1]. One of them would be maintaining lighting quality. During the test, the group of archive users quickly become skillful in finding imperfections of the pictures. Another difficulty arises from the various subjects of photographs and the viewer's different attitudes towards them, which, in turn, would make us deal with a problematic random factor. Therefore, to avoid the aforementioned problems, it is reasonably

*gromaszekm@op.pl

${ }^{\dagger}$ mmichalik@wshe.lodz.pl 
regarded as more beneficial to apply an objective method of evaluation instead. The list of such methods, including their statistic analysis is published in paper [2]. The most commonly used are MSE (mean squared error) and SNR (signal to noise ratio) [3]. The evaluation of digital photographs quality made by these indices is often different from human assessment [5].

Unfortunately, these propositions are not as simple and refined as the indices based on the Minkowski's metric and its derivatives.

Our proposition does not aspire to an universal image quality index. On the contrary - it is designed as a specialist tool for comparison of the monochrome portrait quality. Another issue has also an integral part in the process of digitalizing of archives, that is a correction of scanned images, either as a part of the scanning process or as a separate procedure based on processing previously scanned materials in uncontrolled conditions. By correction we mean for instance eliminating printing raster, sharpening or contrast correction.

Our index is based on the one presented in paper [6]. That index has many excellent features - it reflects precisely the control group's assessment of the images quality. However, our careful research led us to the conclusion that in the case of analyzing monochrome low quality photographs with printing raster traces and with low contrast and noise, that index applies less successfully. In order to improve its efficiency we suggest accentuating a focal point of every picture, and so deflecting the viewer's attention from its imperfections. In the case of portraits the focal point is always the face of the model. Therefore, ay deformations in this area will particularly affect perception. The scheme of our procedure is as follows: we will select an area of a face automatically (as an ellipse/circle ring) and then we will present a criterion for determining the quality index.

\section{Determining the main ellipse}

We will determine the new index criterion in the subsequent paragraphs. First let us show the way of marking ellipse/ circle ring that encloses the model's face. The circle ring will be area of our main interest, and the focal point of the photographs. The review article [7] presents a range of methods of determining human face or skin. In our research we work on monochrome photographs on particular subject. Hence, it seems reasonable to choose the method based on Hough Transform. Paper [8] delineates how the method may encompass the ellipse. Whereas the paper [9] illustrates how to mark an ellipse in pictures of human face. The algorithm included in paper [10] would be an appropriate one as well.

We decided to adapt simplified Hough Transform. We take the following steps: first we find the edges (we use the easy gradient and thresholding, but every edge detection filter will be appropriate, see [11]) and using a simple height and width parameter of the photograph we determine the range of needed circle radius. There is no need to be very precise, so we transform radii in the determined area but including rsteps $>1$ 
stage. Next we find the centre of the circle for each radius value. The centre of the circle is some value (Hough Peaks). We calculate the average of these values and then we determine the centre as an average of the circles' centers that have the Hough Peaks value greater than their average. We calculate the average radius value in the similar manner - after dismissing the values lower than the Hough Peaks average.

\section{The quality index}

The authors in paper [6] proposed:

$$
Q_{j}=\frac{\sigma_{x y}}{\sigma_{x} \sigma_{y}} \frac{2 \bar{x} \bar{y}}{(\bar{x})^{2}(\bar{y})^{2}} \frac{2 \sigma_{x} \sigma_{y}}{\sigma_{x}+\sigma_{y}},
$$

where:

$$
\bar{x}=\frac{1}{N} \sum_{i=1}^{N} x_{i}, \quad \bar{y}=\frac{1}{N} \sum_{i=1}^{N} y_{i} .
$$

It is assumed that the original image is defined by $\mathrm{x}$ values, and the analysed image is defined by y values:

$$
\begin{aligned}
x=\left\{x_{i} \mid i=1 \ldots N\right\}, \quad y=\left\{y_{i} \mid i=1 \ldots N\right\}, & \\
\sigma_{x}^{2} & =\frac{1}{N-1} \sum_{i=1}^{N}\left(x_{i}-\bar{x}\right)^{2}, \\
\sigma_{y}^{2} & =\frac{1}{N-1} \sum_{i=1}^{N}\left(y_{i}-\bar{x}\right)^{2}, \\
\sigma_{x y} & =\frac{1}{N-1}\left(x_{i}-\bar{x}\right)\left(y_{i}-\bar{y}\right) .
\end{aligned}
$$

The particular elements of the first equation are interpreted in the following way: the first element is the correlation coefficient between $\mathrm{x}$ and $\mathrm{y}$, which measures the degree of linear correlation between $\mathrm{x}$ and $\mathrm{y}$. The second element measures how close the mean illumination is between $\mathrm{x}$ and $\mathrm{y}$. The third product element measures how similar the contrasts of the image are. The first product element is from the range [-1,1], the other ones are from the range $[0,1]$. The value 1 is the highest value that is accepted in case of the identical images.

The technique of the value $\mathrm{Q}$ determining is following (that is described in paper [6]): we calculate the value $Q_{j}$ (from the first equation) for the value $\mathrm{BxB}$ squared (in our paper we assumed $\mathrm{B}=4$ ) starting from the left image top and move our square pixel by pixel to the right and bottom until we reach the right bottom corner. We get $\mathrm{M}$ steps and the final result is calculated from: 


$$
Q=\frac{1}{M} \sum_{j=1}^{M} Q_{j}
$$

where $Q_{j}$ is the index value for the chosen square. If we determine index $\mathrm{Q}$ for the photo that was operated with many destructive methods then in some circumstances the values we get will be different from the index based on addressee opinion (MSR). It happens especially in the case of situations when the correction/deformation is made only in the photo fragment. It is also possible in the case of the particular interest of the central photo element (in our case it is the model face).

Let us calculate the index by taking into consideration the particular area determined by the specified ellipse/circle before.

Now we will calculate the index Q:

$$
Q=\sum_{j=1}^{M} P_{j} Q_{j}
$$

The norm that is to sensitize our index to important photo fragments should meet two conditions:

$$
\begin{aligned}
P_{j} & \geq 0 \\
\sum_{j=1}^{M} P_{j} & =1 .
\end{aligned}
$$

Let us introduce the new designations: where $S_{e}$ is the number of pixels included in ellipse that was specified in the first step, $S_{I}$ is the whole number of photo pixels. Let us introduce the norm:

$$
P_{j}= \begin{cases}\frac{1}{s_{I}^{2} X} & \text {,when the middle square, that we determine Qj for, is in the ellipse; } \\ \frac{1}{S_{I} A} & \text {,in the other cases; }\end{cases}
$$

where $\mathrm{X}$ is a real number that controls the sensitivity of our norm to the ellipse points. Coefficient A:

$$
A=\frac{\left(s_{I}-s_{e}\right) s_{I} X}{s_{I}^{2} X-s_{e}} .
$$

The numerator of the above expression is always positive and the number $\mathrm{X}$ has to meet the condition number (8):

$$
X>\frac{s_{e}}{s_{I}^{2}}
$$


It can be checked by direct calculating that there is an ideal norm for our photo that equals (1) the original norm. Let us calculate it for an ideal image (for each i $x_{i}=y_{i}$ ).

$$
\begin{gathered}
Q=\sum_{j=1}^{M} Q_{j}(=1) P_{j} \\
=\sum_{\substack{\text { ellipse's } \\
\text { points }}} P_{j}+\sum_{\substack{\text { points out } \\
\text { the ellipse }}} P_{j}=s_{e} \frac{1}{s_{I}^{2} X}+\left(s_{I}-s_{e}\right) \frac{1}{s_{I} A} .
\end{gathered}
$$

We have $\mathrm{Q}=1$ by simplifying the formula. $\mathrm{X}$ was selected so that:

$$
\frac{\sum_{\text {ellipse's points }} P_{j}}{\sum_{\text {points outside the ellipse }} P j}=4
$$

and the choice of number 4 is arbitrary.

\section{Findings}

We will show the typical results for the monochrome photo of a man. The photo is low quality and has distinct scanning defects (see Fig. A.1). Let us introduce destructive elements: Fig. A.2 with the artifacts got after the strong JPG compression, Fig. A.3 with the blur filter applied in the area outside the face in Fig. A.2, in Fig. A.4 the blur filter was applied only on the face and Fig. A.5 is the original photo with a noise like Gauss, Fig. A.6 is the effect of the median applied outside the head, Fig. A.6 is obtained by applying the median in the head area, Fig. A.8 is the effect of the median applied in the whole picture.

We determined the index value for the original photo and compared with the test group evaluation. The test group were 45 people and they were asked to order the photos from the best one to the worst one. The Table 1 shows the results - sorted in the ascending order by $Q_{m}$ - our new index.

Similar results were obtained for the other monochrome photos. 
Pobrane z czasopisma Annales AI- Informatica http://ai.annales.umcs.pl

Data: 26/04/2023 16:08:41

Table 1. The comparison of the results of $\mathrm{Q}$ coefficient calculated for one photograph distorted in different ways. The ways of distortions are described in the paper. The number of photograph is in the first column, the index from paper [6] is the second column, our proposition is in the third column, the control group assessment is in the fourth column. Fig. A.1 is the original photograph with evaluation 1.

\begin{tabular}{|l|l|l|l|}
\hline $\begin{array}{l}\text { Illustration } \\
\text { number }\end{array}$ & $\begin{array}{l}\text { formula in paper [6] } \\
\text { equation (7) }\end{array}$ & $\begin{array}{l}\text { our proposition } \\
\text { equation (8) }\end{array}$ & MSR \\
\hline 4 & 0.394 & 0.158 & 0.02 \\
\hline 8 & 0.085 & 0.222 & 0.10 \\
\hline 7 & 0.415 & 0.287 & 0.15 \\
\hline 6 & 0.144 & 0.647 & 0.30 \\
\hline 5 & 0.422 & 0.658 & 0.50 \\
\hline 3 & 0.125 & 0.665 & 0.70 \\
\hline 2 & 0.524 & 0.744 & 0.90 \\
\hline 1 & 1.00 & 1.00 & 1.00 \\
\hline
\end{tabular}

\section{Conclusions}

The index we proposed is an easy in use extension of the universal quality images measure. If we compare it and MSR in use for the monochrome photos it is very advantageous. It is much more advantageous than the index $\mathrm{Q}$. We are conscious of the fact that the method we propose is not universal. But in particular situations using our algorithm brings extreme benefits and it can be used with success in the automatic quality evaluation systems. 
Pobrane z czasopisma Annales AI- Informatica http://ai.annales.umcs.pl

Data: 26/04/2023 16:08:41

\section{A Appendix}

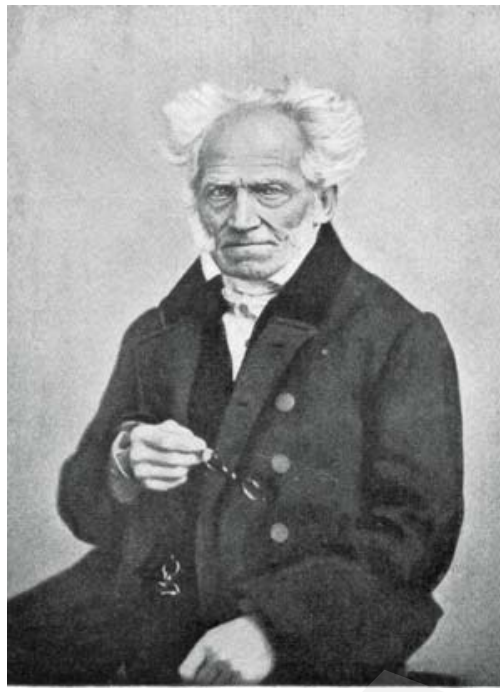

Fig. A.1. The original photograph.

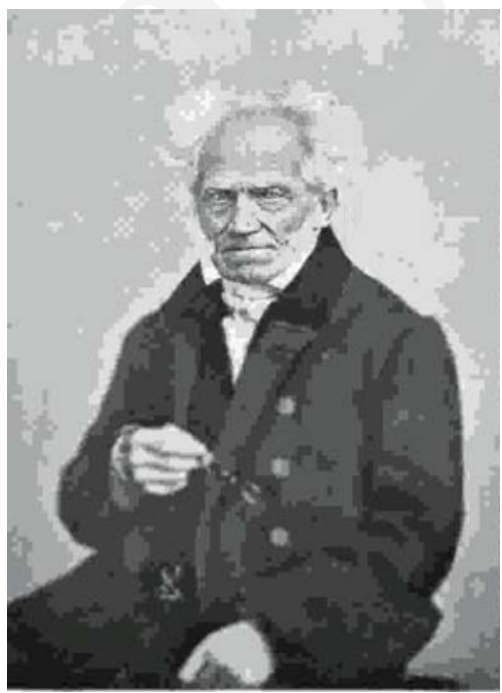

Fig. A.3. The photograph with blur filter in the whole area.

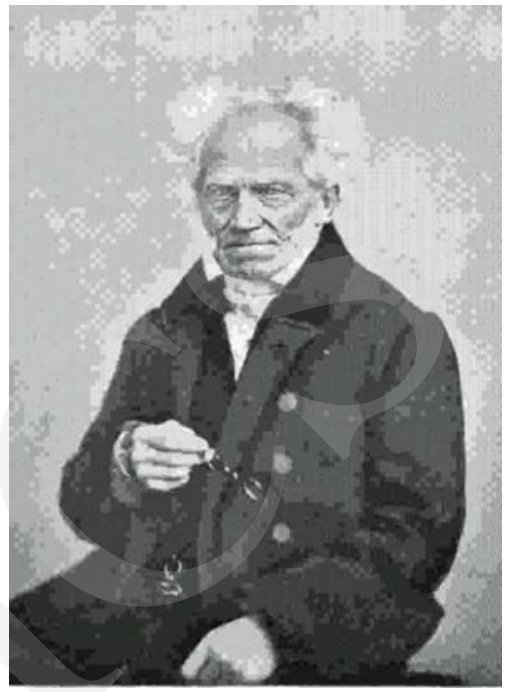

Fig. A.2. The photograph with strong JPG compression.

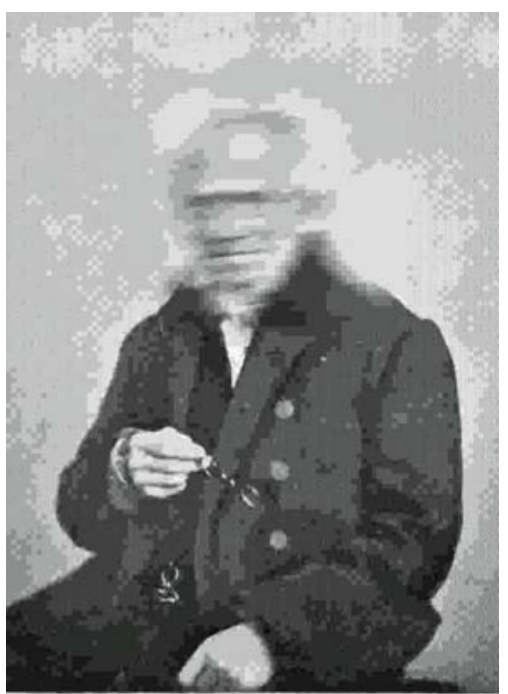

Fig. A.4. Blur filter applied in the face area. 
Pobrane z czasopisma Annales AI- Informatica http://ai.annales.umcs.pl

Data: 26/04/2023 16:08:41

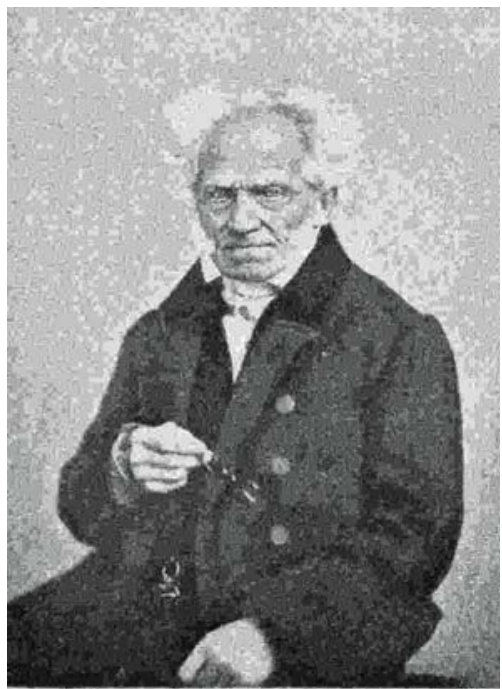

Fig. A.5. The photograph with the Gauss noise.

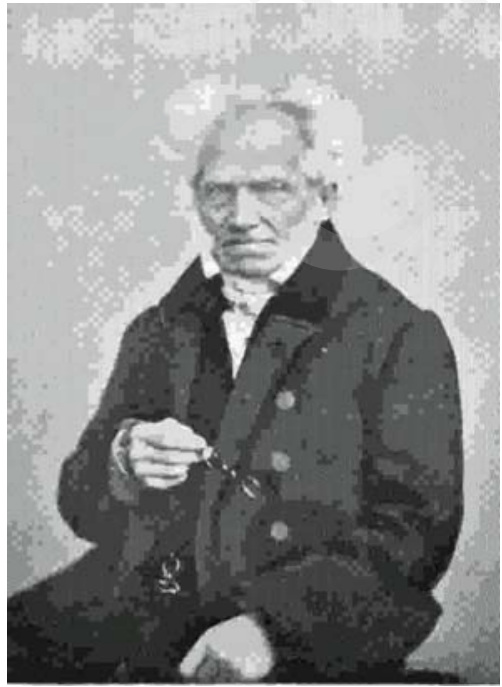

Fig. A.7. Median filter applied in the head area.

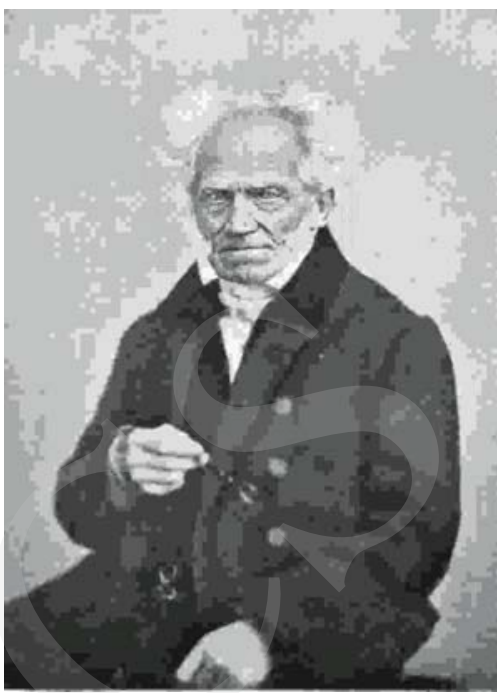

Fig. A.6. Median filter applied in the area outside the head.

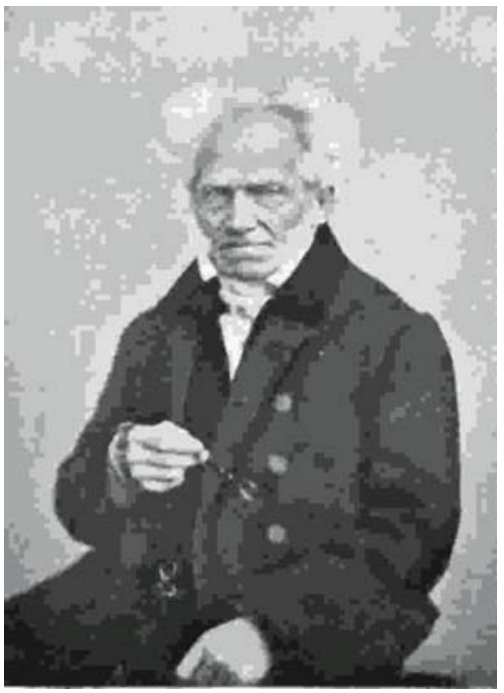

Fig. A.8. Median filter applied in the whole area. 


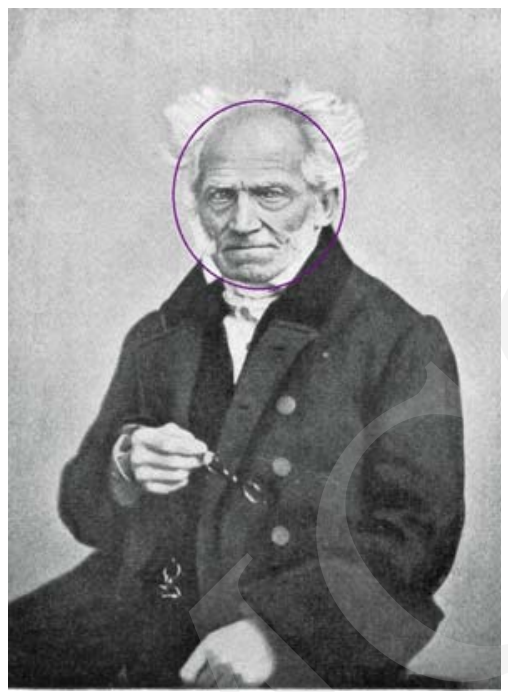

Fig. A.9. The head area marked with the algorithm described in the text.

\section{References}

[1] Richardosn I. E. G., H.264 and MPEG-4 Video Compression (John Wiley and Sons Inc, 2003).

[2] Avcibaş I., Sankur B., Sayood K., Statistical evaluation of image quality measures, Journal of Electronic Imaging, 11(2) (2002): 2006-223.

[3] S. Grgić, M. Grgić, M. Mrak, Reliability of objective picture quality measures, Jornal of Electrical Engineering 55 (2003): 3-10.

[4] Braden C. J., Verscheure L., Verscheure O., Perceptual quality measure using a spatio temporal model of the human visual system, Acoustics, Speech and Signal Processing 4 (1996): 2291-2294.

[5] Wang Z., Bovik A., A universal image quality index, IEEE Signal Proc. Letters XX (2002): 1-6.

[6] Yang M., Kriegman J., Ahuja N., Detecting faces in image: a survey, IEEE Transactions on Pattern Analysis and Machine Intelligence 24 (2002): 34-58.

[7] Aguado A. S., Nixon M. S., A new hough transform mapping for ellipse detection, Research Journal Image Speech and Intelligent Signals 6 (1995).

[8] Prakash J., Rajesh K., Human face detection and segmentation using eigenvalues of covariance matrix, Hough transform and raster scan algorithms, Proc. of Word academy of science, engineering and technology 29 (2008): 372-380. 
Pobrane z czasopisma Annales AI- Informatica http://ai.annales.umcs.pl

Data: 26/04/2023 16:08:41

78

Image quality index of the monochrome archival photographs' compression

[9] Zhang H., Zuo W., Wang K., Zhang D., A Snake-based approach to automate segmentation of tongue image using polar edge detection, International Journal of Imaging Systems and Technology 16 (2006): 103-112.

[10] Pratt W. K., Digital Image Processing (John Wiley and Sons, Inc., 2001). 